\title{
ESTRATEGIAS ARGUMENTATIVAS EN LOS DISCURSOS DIDÁCTICOS DE ARISTÓTELES
}

Claudia SEGGiaro ${ }^{1}$

RESUMEN: En el presente trabajo nos interesa indagar el valor didáctico que el uso de la dialéctica tiene en los escritos aristotélicos. Para ello, lo dividiremos en dos partes. En la primera haremos una somera descripción acerca de la naturaleza de los textos conservados del Estagirita. En la segunda, examinaremos el uso de las estrategias dialécticas empleadas por Aristóteles. Para ello, nos centraremos en obras como Sobre la filosofía, el Protréptico, Metafísica, Tópicos, Analíticos segundos y Ética Nicomáquea, obras en las que hace referencias relativamente explícitas a la cuestión aquí estudiada.

Palabras clave: Aristóteles - dialéctica - didáctica - conocimiento.

ABSTRACT: In this work we examine the didactic value that the use of dialectics has in Aristotelian writings. To do this, we will divide the paper into two parts. In the first one we will make a brief description about the nature of the preserved texts of the Stagirite. In the second, we will examine the use of dialectical strategies used by Aristotle. To do so, we will focus on works such as On Philosophy; Protrepticus, Metaphysics Topics, Posterior Analytics, and Nicomachean Ethics, works in which he makes relatively explicit references to the question studied here.

Keywords: Aristotle - dialectics - didactics - knowledge.

${ }^{1}$ UBA - Conicet. E-mail: claudiasegg@gmail.com

Fecha de recepción: 20/10/2021; fecha de aceptación: 9/12/2021

DOI: https://doi.org/10.46553/sty.30.30.2021.p101-123

Stylos. 2021; 30 (30); pp. 101-123; ISSN: 0327-8859; E-ISSN: 2683-7900 
Solemos leer los textos cuya autoría se adjudica a Aristóteles con el objetivo de reconstruir su pensamiento, ya sea como parte de nuestras investigaciones o para sistematizar las tesis aristotélicas en nuestras clases. Al hacer esto, en general, resaltamos el carácter expositivo de estos textos, quedando en segundo plano el hecho de que muchos de ellos no solo tenían una marcada impronta didáctica, sino que eran las anotaciones o discursos que Aristóteles preparaba para sus lecciones, ya sea en el contexto de la Academia $^{2}$ o en el Liceo. Su objetivo en ellos no era simplemente exponer sus concepciones y persuadir al auditorio sobre su veracidad, sino enseñar e impartir su ideario filosófico a quienes asistían a sus clases. Para ello, Aristóteles apelaba a una serie de recursos calificados de "dialécticos", también utilizados en sus obras exotéricas para dar cuenta de sus concepciones. En función de esto, podemos decir que estos recursos tenían también un innegable un valor didáctico. La apelación y problematización de las creencias admitidas culturalmente o de los pensadores que lo precedieron (Protréptico fragmentos 3132 (Düring); Sobre la filosofía fragmento 17 (Ross), Física I, 2-6), el uso de las analogías (Protréptico fragmentos 11-13; Física II 1, 193a 28-33; Metafísica VII 7 y 8) y de las metáforas (Metafísica II 1, 993b9- 11; fragmento 105 del Protréptico (Düring) Retórica 1410b15) forman parte de estos recursos que suponen un intercambio entre maestro y alumno, cimentada en la relación enseñanza-aprendizaje.

Teniendo esto como telón de fondo, en el presente trabajo nos interesa indagar ese valor didáctico que el uso de la dialéctica tiene en los escritos aristotélicos. Para ello, lo dividiremos en dos partes. Debido a que, para realizar nuestro análisis nos basaremos en algunas de las llamadas obras exotéricas, como, por ejemplo, Sobre la filosofía y el Protréptico, y en algunos de sus discursos escolares, especialmente Metafísica, Tópicos, Analíticos segundos y Ética Nicomáquea, en la primera parte haremos una somera descripción acerca de la naturaleza de los textos aristotélicos y las problemáticas asociadas con ellos. En la segunda, examinaremos el valor didáctico de las estrategias dialécticas utilizadas por Aristóteles en sus discursos.

\footnotetext{
${ }^{2}$ cf. BERTI (1997: 95 ss.).
} 


\section{NATURALEZA Y TRANSMISIÓN DE LOS TEXTOS DE ARISTÓTELES}

Al estudiar el pensamiento de Aristóteles, solemos clasificar sus "escritos" en dos grandes grupos: las "lecciones escolares" u obras acroamáticas y los discursos públicos. Esto implica al menos dos problemáticas. La primera alude al alcance de la distinción propiamente dicha. La segunda problemática, vinculada con esta, es en qué medida el examen conjunto de ambos tipos de obras nos permite reconstruir el pensamiento del Estagirita de una manera más íntegra que el análisis centrado solo en sus llamadas lecciones de clase.

Si bien Aristóteles hace referencia a sus exoterikoí lógoi en sus lecciones, ${ }^{3}$ debido a que, hasta la aparición de la edición de Andrónico de Rodas, estas circulaban de manera restringida ${ }^{4}$ y que los primeros se conservan

${ }^{3}$ VALLEjo CAMPOS (2005) recoge ocho referencias de Aristóteles a sus "obras exotéricas". Entre ellas, podemos mencionar: Ética Nicomáquea VI 4, 1140a1-3; Ética Eudemia I 8, 1217b19-23; II 1, 1218b32-34; Metafísica XIII 1, 1076a26-29, Política III 6, 1278b30-32; VII 1,1323a21-23; Física IV 10, 217b29-32.

${ }^{4}$ Según Düring (2005 [1966]: 90), en sus cartas Epicuro habla de los Analíticos y de la Física. Para este autor, los fragmentos de Sobre la Naturaleza son una prueba de que este pensador estaba familiarizado con Sobre el cielo y que probablemente conoció alguno de los textos éticos. Desde su punto de vista, esto es una evidencia de que Teofrastro tuvo que haber redactado o haber hecho redactar algunos de los escritos de Aristóteles y hacerlos asequibles en el mercado. TANNER (2010: 125), por su parte, señala que Posidonio de Apamea tuvo acceso a algunos tratados aristotélicos durante la época en que se suponía que estaban perdidos. Para este autor, fuentes antiguas atribuyen explícitamente a Posidonio el uso directo de Sobre el alma y Sobre el cielo, mientras que los fragmentos conservados de su obra permiten constatar que también usó la Meteorología. Paralelamente, HatzimichaLi (2012: 15) afirma que Estrabón habla de la producción de copias de las obras de Aristóteles como una actividad comercial y, por tanto, potencialmente lucrativa. Esto último se habría llevado, según Hatzimichali, en el momento en que Tiranión estaba realizando una edición de los manuscritos aristotélicos. En la misma línea, PAJÓN LEYRA (2013: 724) argumenta que hay evidencias de que en el período helenístico existía cierta circulación de varias obras aristotélicas en los diversos centros culturales asociados a los peripatéticos. Quizá por esto, HAtzimichali (2013: 17) concluye que, aunque es poco probable que las obras de Aristóteles y Teofrasto fueran en ese momento ampliamente conocidas y estuviesen completamente disponibles, la idea de que se conservó solo una copia difícilmente pueda sostenerse; por lo que el problema no es determinar si hubo una pérdida total del corpus, sino cuándo y dónde se conocieron los diferentes tratados, y cuál es su relación con la versión del corpus que disponemos hoy. Un trabajo que es central para

Stylos. 2021; 30 (30); pp. 101-123; ISSN: 0327-8859; E-ISSN: 2683-7900 
fragmentariamente, históricamente fue un problema establecer qué tipo de diferencias existían entre ambos tipos de discursos. Vallejo Campos ${ }^{5}$ destaca que, tanto para Cicerón, ${ }^{6}$ como para los neoplatónicos, los lógoi exoterikoí sólo se distinguían de los acroamáticos por su destinatario y por su estilo literario más depurado y adaptado al público al que iban dirigidos. De hecho, señala Düring, ${ }^{7}$ pensadores, como, por ejemplo, Amonio (Comentario a las Categorías de Aristóteles 4, 5-27), Olimpiodoro (prolegómenos a las Categorías de Aristóteles 7, 3-21) y Elías (Comentario a las Categorías de Aristóteles, 115, 3-5) corrigieron la interpretación de Alejandro de Afrodisia, quien suponía que en sus lecciones u obras acroamáticas Aristóteles exponía sus propios puntos de vista y la verdad, mientras que en los discursos exotéricos reproducía opiniones que no eran propias (Elías, Comentario a las $\mathrm{Ca}$ tegorías de Aristóteles, 115, 3-5), ${ }^{8}$ valiéndose para ello de diversos interlocutores. $^{9}$ Jaeger (1993[1923]) atribuye esta distinción trazada por Alejandro no solo al hecho de que el pensamiento de Aristóteles habría atravesado por una evolución que comenzó por una fase platónica, sino a que la mayor par-

el análisis de esta cuestión es "Roman Aristotle" de BARNES (1997: 1- 70). Hatzimichali, acuerda con este autor que no hay base para sostener que Andrónico de Rodas fue el primer editor de la obra aristotélica y que esta no estuvo disponible hasta la aparición de dicha edición. Pese a ello, en oposición a Barnes, considera que el pronunciamiento de Andrónico sobre la naturaleza de los escritos, sobre todo en lo que respecta a su autenticidad, y su trabajo de ordenación transformaron el modo en que se leyó posteriores a Aristóteles y opacó las obras más conocida y ampliamente disponibles durante la vida de su autor: los exoterikoi lógoi.

${ }^{5}$ VALLEJO CAMPOS (2005: 33).

${ }^{6}$ La descripción que Cicerón realiza de las obras aristotélicas vuelve plausible pensar que debió haber conocido tanto los textos exotéricos como algunos esotéricos. En Tópicos I,3, destaca la oscuridad y la dificultad de ciertas obras aristotélicas en una clara alusión a los trabajos esotéricos. En Del supremo bien y del supremo mal V 5, 12 resalta que Aristóteles tenía dos tipos de textos: "los escritos en un estilo popular, que denominaban exotéricos, y otros en un estilo más elaborado, que dejaron en forma de comentarios, no siempre parecen decir lo mismo. Sin embargo, en general, no hay divergencia alguna entre éstos, al menos entre los que he citado, ni desacuerdo en ellos mismos" (trad. Vallejo Campos).

${ }^{7}$ DÜRING (1957: 437).

${ }^{8}$ Cf. DÜRING (1957: 437-440).

${ }^{9}$ DüRING (1957: 437) conjetura que Alejandro de Afrodisia habría realizado esta distinción en el comentario perdido de Acerca del alma.

Stylos. 2021; 30 (30); pp. 101-123; ISSN: 0327-8859; E-ISSN: 2683-7900 
te de su producción filosófica de ese período se habría plasmado en sus obras exotéricas. Para este autor, el cambio doctrinal que se podría trazar entre muchos de estos discursos y las lecciones esotéricas fue la causa de que este pensador no supiera cómo catalogar a los escritos exotéricos o los tomara como "fuentes de un platonismo puro". ${ }^{10}$ A juicio de Jaeger, en aquel tiempo se consideraba que había contradicciones entre los dos géneros de discursos. "Los esfuerzos de los peripatéticos posteriores para explicar este embarazoso estado del asunto pueden descubrirse en la conocida tradición acerca de la diferencia entre los escritos exotéricos y los esotéricos. Los estudiosos buscaban naturalmente la explicación de los diálogos en los tratados, y la encontraban en la frase 'discursos exotéricos', que aparece varias veces y en algunos casos puede referirse fácilmente a los diálogos publicados. En oposición a estos discursos exotéricos o dirigidos al mundo exterior, consideraban los tratados como un cuerpo de doctrina esotérica o secreta, aunque no hay indicio alguno de semejante noción o expresión en Aristoteles". ${ }^{11}$ Desde la perspectiva de Jaeger, los testimonios de algunos pensadores, como Plutarco, Proclo y Diógenes, habrían hecho difícil ver la evolución que sufrió el pensamiento aristotélico en la Antigüedad, alimentado la teoría de la ruptura entre ambos tipos de obras.

Partiendo de la misma tesis evolucionista de Jaeger, Bignone ${ }^{12}$ retoma la distinción de Alejandro de Afrodisia, no para refutarla, sino para corregirla. Para Bignone, al igual que para Alejandro de Afrodisia, la distinción entre ambos tipos de discursos: exotéricos y esotéricos, no era solo de forma, sino de contenido. Sin embargo, a diferencia del peripatético, este autor considera que las concepciones de raigambre platónicas supuestamente presente en los discursos exotéricos y cuestionadas posteriormente en sus lecciones de clases, habrían sido defendidas por Aristóteles en el período en que estos fueron elaborados. Para Bignone, ${ }^{13}$ la conciencia de esta diferencia entre las obras publicadas, de carácter platónico, y los escritos escolares, netamente aristotélicos, era muy profunda en la época imperial. Una de las pruebas de

\footnotetext{
${ }^{10}$ JAEGER (1993[1923]: 44).

${ }^{11}$ JAEGER (1993[1923]: 44-45).

12 BigNONE (1973)

${ }^{13}$ BignONE (1973: 179).
}

Stylos. 2021; 30 (30); pp. 101-123; ISSN: 0327-8859; E-ISSN: 2683-7900 
esto era, según él, el testimonio de Luciano de Samosata, quien sostenía que había dos Aristóteles diferentes en una sola persona: uno esotérico y el otro exotérico (Vit. Auct. 26).

Compartimos con Düring, ${ }^{14}$ que esta posición es insostenible. En primer lugar, porque la lectura de los textos conservados del Estagirita, sean estos exotéricos o esotéricos, vuelve inviable defender la existencia de un período netamente platónico, como parece creer este autor. ${ }^{15}$ En segundo lugar, porque la supuesta evolución de un platonismo a un aristotelismo no parece ser un criterio viable para diferenciar a los discursos aquí analizados. De hecho, Plutarco refuta ambas cosas al afirmar que:

"En lo que se refiere a las ideas, respecto a las cuales critica a Platón, Aristóteles, al cuestionarlas en todas partes y suscitar todo tipo de objeciones contra ellas en sus tratados éticos, (metafísicos) y físicos y en sus diálogos exotéricos, pareció a algunos que se dejaba llevar más por el afán de polémica que por motivos filosóficos, como si su intención fuera despreciar la filosofía de Platón57. Hasta tal punto estaba lejos de seguirla". (Plutarco Contra Colotes, 447F448A trad. Vallejo Campos).

\footnotetext{
${ }^{14}$ DÜRING (2005 [1966]: 66).

${ }^{15}$ En relación con esto, se debe señalar que aun en el Protréptico, que es la obra en la cual los partidarios de la tesis evolucionista han encontrado más apoyo textual, esta tesis resulta problemática. Para esta cuestión, cf. SegGiaro (2016). La tesis de este artículo es que quienes creen que Aristóteles defendía en el Protréptico la teoría de las Ideas caen en la misma crítica que el Estagirita realiza a los platónicos, cuando estos justifican la existencia de principios trascendentes apelando al argumento de la ciencia. En ambos casos, concluyen más de lo que el texto permite concluir. Esto se debe básicamente a que quienes sostienen que hay presencia de la teoría de las Ideas en el Protréptico basan su tesis en que en este texto Aristóteles habla de "lo que es en sí mismo" y de imitaciones; cf. el frag. 47-50 (Düring). Al hacer eso, pierden de vista que también Aristóteles usa las mismas expresiones y los mismos términos para presentar sus concepciones en las obras no calificadas de platónicas. En los escritos éticos promulga la existencia de un bien en sentido absoluto o el bien en sí (Ética Eudemia I 8, 1217b 3-5), en la Metafísica alude explícitamente a la ousía como aquello que es por sí (Metafísica VII 4 1029b) y, paralelamente, se refiere a las técnicas como aquello que imita la naturaleza (Física II 1, II 8). Dado esto, presuponer sobre la base del uso terminológico que Aristóteles sostiene la teoría de las Ideas es pretender que el texto diga más de lo que por sí mismo dice.
}

Stylos. 2021; 30 (30); pp. 101-123; ISSN: 0327-8859; E-ISSN: 2683-7900 
Sobre la base de los testimonios, algunos provenientes de Aristóteles (Ética Eudemia 1 8, 1217b22-23), Jaeger ${ }^{16}$ y Vallejo Campos, ${ }^{17}$ argumentaron que la diferencia entre las discusiones exotéricas (exoterikoi lógoi) y filosóficas (katà philosophían) radicaba en que las primeras habían llegado al público, ${ }^{18}$ mientras que las segundas eran las lecciones regulares que se dictaban en su escuela, esto es, "las bases escritas de sus bastas actividades como profesor y conferenciante". ${ }^{19}$ Jaeger es enfático al sostener que la diferencia central entre ambos tipos de discursos no estaba relacionada ni con el destinatario, ni con la forma de exponer las tesis defendidas o con la naturaleza de las doctrinas presentadas, sino entre si estos podían recibir el calificativo "publicado" o "no publicado".

Retomando parcialmente esta interpretación, Düring ${ }^{20}$ y Vallejo Campos ${ }^{21}{ }^{1}$ llaman la atención sobre el alcance que tenía el hecho de publicar un texto en el siglo IV a. C., pues "el público al que se dirigían los filósofos en este tiempo podía estar constituido por un círculo muy limitado de discípulos". ${ }^{22}$ Hatzimichali ${ }^{23}$ agrega que "publicar un libro" en la Antigüedad podía significar colocar los textos en algún lugar donde estuviesen disponibles para copiar o la lectura pública de un determinado contenido. De hecho, tal como lo recalca Vallejo Campos, Aristóteles califica a estas obras como "lógoi". Ahora bien, debido a su amplitud semántica, mediante el uso de este término Aristóteles podría estar haciendo referencia "a debates, conversaciones o "puntos de vista generalmente conocidos". ${ }^{24}$ Por este motivo, es posible que a través de la expresión exoterikoi lógoi Aristóteles esté pensando en discusiones públicas y no solamente en obras escritas. Esto no quita

${ }^{16}$ JAEGER (1993[1923]: 294-295).

17 VALLEJO CAMPOS (2005: 29- 30).

18 Véase también LloYd (1968: 10). Para este autor, la palabra 'exotérico' puede haber sido usada para calificar a ciertos discursos/obras que estaban al alcance de un público que no pertenecía al círculo de los discípulos de Aristóteles.

${ }^{19}$ JAEGER (1993[1923]: 35).

${ }^{20}$ DÜRING (1957: 442).

${ }^{21}$ VALLEJO CAMPOS (2005: 30).

22 VALLEJO CAMPOS (2005: 30).

${ }^{23}$ HATZimichali (2012: 15).

${ }^{24}$ VALlejo CAMPOS (2005: 30).

Stylos. 2021; 30 (30); pp. 101-123; ISSN: 0327-8859; E-ISSN: 2683-7900 
que, tal como lo evidencian los testimonios que nos han llegado, los discursos exotéricos hubiesen tenido un estilo más cuidado y depurado que el resto de las obras no "públicas". Según Aulo Gelio:

"Aristóteles dedicaba las mañanas a impartir en el Liceo esta enseñanza que he llamado "acroamática" y no admitía en estas sesiones a cualquiera al azar sino a aquellos cuya inteligencia hubiese examinado previamente, así como su formación elemental, su afán de aprender y su trabajo. Pero las lecciones exotéricas y las prácticas de elocuencia las daba en el mismo lugar por la tarde y admitía a ellas a todos los jóvenes sin distinción alguna". (Noches áticas XX 5, trad. Vallejo Campos)

Si bien en las líneas precedentes Aulo Gelio, sostiene que en los discursos exotéricos, a diferencia de las esotéricos, Aristóteles discute cuestiones sobre retórica, ética y política, el análisis de los fragmentos del Protréptico, de Sobre la filosofía, de Sobre las Ideas y de algunos escritos lógicos muestra que esta afirmación no es completamente certera. Una lectura de estas obras nos permite encontrar en ellas muchas de las concepciones metafísicas, gnoseológicas, ético- políticas y metodológicas de las lecciones escolares. La noción de phýsis, central en Física II, es fundamental en el Protréptico (fragmentos 11-17 Düring); la identificación entre epistéme y el conocimiento de los primeros principios no solo aparece en la Metafísica sino en Sobre la filosofía (fragmento 8b- 8c (Ross) y en el Protréptico (fragmentos 34-37 Düring); la identificación de la felicidad con la vida contemplativa de Ética Nicomáquea X aparece replicada en el Protréptico (fragmento 4244 Düring) y las funciones educativas de la poética y de la música sistematizadas en Política VII- VIII son abordadas en Sobre los poetas (fragmentos 5a-b Rose) y el Político (fragmentos 3a-f Rose). Así mismo, se incluye entre los escritos exotéricos algunos títulos y fragmentos de obras en las que Aristóteles habría abordado cuestiones metodológicas: Sobre los problemas, Divisiones, Sobre los contrarios, etc. 
Por algunos testimonios sabemos que algunos de estos discursos exotéricos eran diálogos ${ }^{25}$ que fueron escritos o pronunciados en el período de pertenencia de Aristóteles en la Academia. No obstante, también se presume que en esa época Aristóteles impartía algunos cursos, algunos de ellos de retórica, ${ }^{26}$ en los cuales exponía las tesis de sus obras públicas. Dado esto, parafraseando a Moraux, ${ }^{27}$ podemos decir que todos los textos conservados del Estagirita, incluso los exotéricos, estaban vinculados con su actividad docente. Por tal motivo, pueden verse como una prolongación de esta función que signó su quehacer filosófico desde sus inicios.

Ahora bien, los testimonios conservados son una evidencia relativamente contundente de que, tanto en sus discursos exotéricos, como en sus lecciones de clase, en el orden de la exposición Aristóteles apela a las mismas estrategias argumentativas, entre ellas, las utilizadas en la dialéctica. ${ }^{28}$ Esto nos permite hablar de una implementación didáctica de estas estrategias, cuyo objetivo era conducir el proceso de enseñanza siguiendo el orden natural en el cual se produce el aprendizaje. En la segunda parte de nuestro trabajo nos detendremos a demostrar esto último.

\footnotetext{
${ }^{25}$ Según JAEGER (1993 [1923]: 39), todos los miembros de la Academia escribieron diálogos. Sin embargo, sostiene este autor, al adoptar este género literario, Aristóteles realizó innovaciones significativas, iniciando lo que se podría calificar como "diálogo de discusión científica" (JAEGER,1993 [1923]: 39- 42). Este tipo de dialogo se caracterizaba por ser un largo examen en torno a tu tema, mediante un método riguroso.

${ }^{26}$ BERTI (1997: 95 ss), compara la publicación de Grillo, una de las primeras obras de Aristóteles, con lo que, en un sentido moderno, se podría calificar "dissertazione di libera docen$z a$ ". Berti, señala que poco después de su publicación, Aristóteles dictó en la Academia un "curso" de retórica, en el cual se plasmaban las tesis de esta obra.

${ }^{27}$ Moraux (1951: 10).

${ }^{28}$ Véase Cicerón Del supremo bien y del supremo mal V 4, 10- 1 1; Aulo Gelio, noches áticas XX 5; Elías, Com. de las «Categorías» de Aristóteles 124, 3-6; Quintiliano Inst. orat. II 17, 1-14. La implementación de la dialéctica en las obras exotéricas es evidente en la serie de fragmentos 2a-f (Ross); 53 (Düring) del Protréptico, en los fragmentos 16, 17, 19a-c (Ross) de Sobre la filosofía, en los pocos fragmentos conservados de Sobre la nobleza y en los fragmentos 5a-c de Sobre los poetas (Ross).
}

Stylos. 2021; 30 (30); pp. 101-123; ISSN: 0327-8859; E-ISSN: 2683-7900 


\section{LOS PROCEDIMIENTOS DIALÉCTICOS AL SERVICIO DE LA DIDÁCTICA}

En el fragmento 15b de Sobre la filosofía (Pselo, Escolios a J. Climaco), Aristóteles diferencia el discurso didáctico de la iniciación mistérica. Desde su perspectiva, mientras esta última es una experiencia intelectiva, que reviste la forma de una iluminación, el primero se oye. Esto presupone, una diferencia ulterior, pues, la iniciación mistérica es una vivencia particular y, por tal motivo, intransferible, mientras que el discurso didáctico supone la trasmisión de un determinado saber y, en este sentido, una relación entre al menos dos individuos. En Retórica I 5, 1355a24-29, completa esta descripción al vincular el discurso didáctico con el científico y aquel que se vale de la ciencia más exacta para persuadir al auditorio sobre una determinada tesis. En Tópicos VI 4, 141a28-31, afirma:

"En efecto, cuando la definición se da en vista a conocer lo definido, no conocemos a partir de cualquier cosa, sino a partir de cosas anteriores y más conocidas, tal como en las demostraciones (en efecto, así procede toda enseñanza y todo aprendizaje

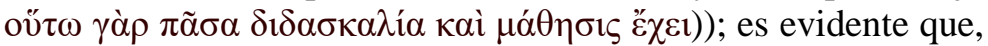
el que no define mediante tales cosas, no ha definido" (trad. Candel Sanmartín).

En este pasaje, en el contexto del análisis de la definición y de su papel epistémico Aristóteles sistematiza la distinción entre lo más conocido en sí y lo más conocido para nosotros. ${ }^{29}$ Allí, sostiene que la definición debe enunciarse sobre la de lo que es anterior en sí o por naturaleza y no en función de lo que es anterior para nosotros. ${ }^{30}$ La causa de esto estriba en que "lo anterior para nosotros" suele identificarse con las características sensibles (Tópicos VI 4, 141b 10) y, por lo tanto, accidentales de las cosas, mientras que "lo anterior en sí" es la esencia o configuración conceptual de esas cosas

\footnotetext{
${ }^{29}$ Cf. Cleary (1997).

${ }^{30}$ Cf. Bronstein (2016), Charles (2010).
} 
(Protréptico frags. 35; 47-48 (Düring)), razón por la cual aporta verdadero conocimiento (Metafísica I 1, 981b 10-19, Sobre la filosofía frag. 8b Ross).

Sin embargo, lo relevante a los fines de nuestro trabajo es que partir de lo más conocido en sí es, según Aristóteles, el modo de proceder en la enseñanza (didaskalía) y en el aprendizaje (máthesis), pues, desde su perspectiva, la posibilidad de enseñar (dúnasthai didáskein) es lo distintivo del que sabe frente al que no sabe (Metafísica I 1, 981b 6). Ahora bien, esto es lo propio del que conoce las causas y, por lo tanto, del que posee epistéme y no empeiría. Quizá por esto Rapp ${ }^{31}$ asevera que el proceso didáctico es estructuralmente similar a la prueba científica (apódeixis). ${ }^{32}$ Esto significa que el profesor ofrece ciertas premisas y conclusiones y que el alumno ha adquirido el conocimiento en cuestión no solo porque lo entiende, sino porque además comprende cómo la conclusión se desprende de las premisas dadas. Sobre la base de tópicos VIII 5, 159a28-30 y Refutaciones sofísticas 2 165b1-3, Rapp argumenta que en este proceso de aprendizaje es importante que el alumno asuma que las premisas transmitidas son verdaderas, incluso si todavía no es capaz de comprender por qué lo son y qué explican.

Con Rapp, podemos afirmar que es cierto que Aristóteles considera que en el orden del conocimiento se debe proceder demostrativamente, tomando como punto de partida los principios y causas, para a partir de ellos obtener el resto del conocimiento (Analíticos segundos I 2, Física I 1, 184a10-15). Dado esto, si el discurso didáctico debe adoptar el modo por el cual conocemos, este debería ser demostrativo. Sin embargo, reducir el discurso didáctico a esto deja sin explicar por qué en sus lecciones: Metafísica I; IV, Física I, Acerca de la generación y corrupción, por ejemplo, Aristóteles se detiene en el análisis de esos principios; es decir, no da cuenta de cuál sería el status de muchos de los discursos de Aristóteles que, tal como lo vimos en la primera parte, eran también notas de clase.

Así mismo es relevante remarcar que Aristóteles no solo no descarta que existan otras formas de conocer (Analíticos segundos I 2, 71b16), sino que admite que el arriba señalado no es el modo natural de hacerlo para la mayoría, sino de alguien que tiene un pensamiento riguroso y excepcional

${ }^{31}$ RAPP (2009: 594).

${ }^{32}$ Analítico posteriores I 2, 71b9-15.

Stylos. 2021; 30 (30); pp. 101-123; ISSN: 0327-8859; E-ISSN: 2683-7900 
(Tópicos VI 4, 141b12-14). Desde su perspectiva, es mejor conocer lo posterior mediante lo anterior en sí, pues tal proceder es más rico en conocimiento. No obstante, para los que no tienen la capacidad de conocer de este modo, o para los que aún no conocen los primeros principios, es necesario hacerlo mediante las cosas conocidas para ellos (Tópicos VI 4, 141b9-19). Por tal motivo, las lecciones del maestro deberían adoptar también esta modalidad. La causa de esto es que:

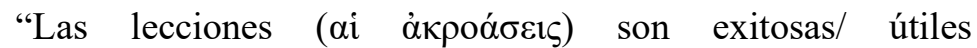

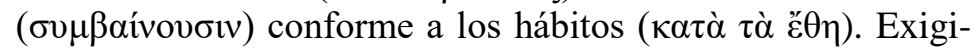
mos, desde luego, que las cosas se digan como estamos habituados ( $\varepsilon i \omega \dot{\theta} \theta \alpha \mu v)$, y las que se dicen de otra manera no pare-

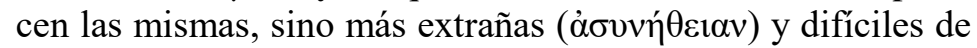
conocer ( $\dot{\alpha} \gamma \omega \omega \sigma \tau o ́ \tau \varepsilon \rho \alpha)$, al no ser habituales. Y es que lo habitual, en efecto, es más fácilmente cognoscible" (Metafísica II 3, 994b32- 995a3. Trad. Calvo Martínez levemente modificada).

Como en muchas de sus obras, en este pasaje de la Metafísica Aristóteles realiza algunas precisiones metodológicas. En este caso en particular, se centra en aquello que es importante en relación con las lecciones. Si bien las cuestiones allí abordadas son tratadas en Física I, 184a16-23; Metafísica VII 3, 1029a 1-4; Analíticos segundos I 2, 71 b33 y ss, Tópicos VI 4, 142a612, Sobre la filosofía fragmento 8b (Ross), Protréptico fragmentos 102, 105 (Düring), 10a2, (Rose), en todos estos pasajes Aristóteles realiza una distinción teniendo en cuenta fundamentalmente el objeto de conocimiento y el grado de dificultad que tenemos para acceder a él. Por tal motivo, podemos decir que en esas instancias Aristóteles explicita el orden natural en el cual conocemos. En el pasaje citado de la Metafísica, en cambio, está reflexión es funcional a cómo debemos actuar en el proceso de enseñanza. ${ }^{33}$ Para Aristó-

${ }^{33}$ En Analíticos segundos I 1, Aristóteles arranca su argumento sosteniendo que toda ense-

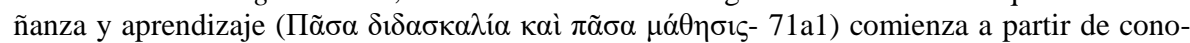
cimientos preexistentes. En relación con esto, sostiene que, cuando este proceso de enseñanza y aprendizaje se da a partir de razonamiento, el punto de partida es aquello previamente acor- 
teles, lo más habitual es en términos cognitivos lo más cognoscible para nosotros. De las obras mencionadas más arriba se desprende que el proceso natural del conocimiento y, por ende, del aprendizaje es partir de esto último, para alcanzar lo más cognoscible en sí, esto es, los principios y causas propios de cada ámbito de estudio. De esto se desprende que, para que la lesión cumpla su fin, se debe poner en marcha este proceso por el cual naturalmente aprendemos. Ahora bien, para que esto suceda, esta debe pronunciarse de modo tal que lo dicho resulte habitual, es decir, sea conforme a nuestros hábitos. ${ }^{34} \mathrm{El}$ uso del circunstancial "katà tà éthe" es clave, porque indica que no solo el lenguaje utilizado debe adecuarse a lo que el auditorio puede entender, sino que las estrategias empleadas para trasmitir el contenido también se deben ajustar a los hábitos del público; pues lo que no es dicho de este modo, resulta oscuro y extraño y, por lo tanto, difícil de conocer.

Para esto, Aristóteles acude a ciertas estrategias argumentativas que son propias de la dialéctica. Para Aristóteles, si bien el discurso dialéctico difiere del didáctico ${ }^{35}$, esto no implica que muchos de los recursos utilizados en los primeros no sean útiles en el orden la de explicación y del conocimiento. Desde su perspectiva, la dialéctica es útil para la filosofía,

"porque, pudiendo desarrollar una dificultad en ambos sentidos, discerniremos más fácilmente lo verdadero y lo falso en cada cosa. Pero es que además es útil para las cuestiones primordiales propias de cada conocimiento. En efecto, a partir de lo exclusivo de los principios internos al conocimiento en cuestión, es imposible decir nada sobre ellos mismos, puesto que los

dado. Con esto último Aristóteles podría estar haciendo referencia a los éndoxa o las opiniones que son apoyadas por la mayoría, por los sabios o por la mayor parte de los sabios, esto es, por lo más conocido para nosotros.

${ }^{34} \mathrm{cf}$. Alejandro de Afrodisia, Comentario a la Metafísica de Aristóteles, 167, 5 ss.

${ }^{35}$ En Refutaciones sofísticas en el contexto de la clasificación de los cuatro géneros de discursos, Aristóteles distingue entre aquellos que son didácticos y los que son dialécticos. La diferencia entre ambos tipos de discursos es que los primeros "prueban los que prueban a partir de los principios peculiares de cada disciplina y no a partir de las opiniones del que responde (pues es preciso que el discípulo se convenza)" (Refutaciones sofísticas 165b1-3), mientras que los segundos "prueban la contradicción a partir de cosas plausibles" (Refutaciones sofísticas $165 \mathrm{~b} 5$. Trad. Candel Sanmartín).

Stylos. 2021; 30 (30); pp. 101-123; ISSN: 0327-8859; E-ISSN: 2683-7900 
principios son primeros con respecto a todas las cosas, y por ello es necesario discurrir en torno a ellos a través de las cosas plausibles concernientes a cada uno de ellos. Ahora bien, esto es propio o exclusivo de la dialéctica: en efecto, al ser adecuada para examinar (cualquier cosa), abre camino a los principios de todos los métodos (Tópicos 101a33- 101b4. Trad. Candel Sanmartín)

La lectura de este pasaje pone en evidencia que hay al menos dos motivos, ambos relacionados, por los cuales la dialéctica es relevante en los procesos didácticos. El primero está vinculado con la naturaleza de los principios: puesto que no puede haber demostración en sentido estricto de estos principios (Analíticos segundos I 2), la dialéctica es el único modo de dar cuenta de ellos. En este sentido, la dialéctica es la base para que posteriormente la enseñanza pueda seguir el curso de una investigación científica, tal como la describió Rapp en el trabajo comentado más arriba. El segundo motivo es que la dialéctica parte de premisas plausibles. Esas premisas son aquellas creencias u opiniones que desde el punto de vista epistémico son lo más conocido para nosotros. Dado esto, se puede decir que la dialéctica permite aprender algo, siguiendo el orden natural en el que solemos conocer, garantizando, en este sentido, la utilidad o el éxito de las lecciones. (Düring):

Este modo de proceder es explícito en el fragmento 31 del Protréptico

"Además, puesto que todos preferimos cuanto es posible y provechoso, hay que mostrar que tanto lo uno como lo otro corresponde al filosofar, y que la dificultad de su adquisición es inferior a la magnitud de su provecho; pues todos hacemos lo más fácil con mayor placer" (trad. Vallejo Campos).

En esta obra, al igual que en la Ética Nicomáquea, Aristóteles formula su ideario filosófico que identifica el fin último del hombre con la realización de su función propia y a esto último con la actividad contemplativa. Para ello, parte de un éndoxon que ningún hombre pondría en cuestión: la persecución de lo útil y fácil de realizar. El objetivo de Aristóteles es demostrar 
que la filosofía permite al hombre alcanzar dicho fin, identificado con la felicidad, y que cumple ambos requisitos: ser útil y fácil. El motivo por el cual Aristóteles se ve obligado a realizar esta demostración es que, si bien reconoce que todos los hombres buscan la felicidad y tienden a hacer aquello que es útil, también se percataba de que no hay un acuerdo sobre qué cosas proveen la felicidad y cuál es el criterio adecuado para establecer grados de utilidad. Por otra parte, es consciente de que la mayoría de los hombres consideran que la filosofía carece de utilidad. ${ }^{36}$ Argumentativamente, una de las estrategias empleadas por Aristóteles en este texto es demostrar la inconsistencia que existe entre defender que el fin último del hombre es la felicidad y la negativa de los hombres a filosofar. El modus operandi implementado para lograr esto no aparece en los fragmentos conservados del Protréptico, pero es descripto en algunas de sus otras obras, como, por ejemplo, la Ética Nicomáquea:

"como en los demás casos $<$, dice Aristóteles, $>$ después de es-

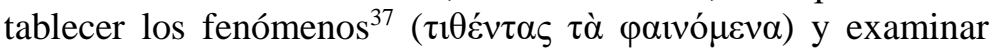
las aporías ( $\delta 1 \alpha \pi \circ \rho \eta ́ \sigma \alpha v \tau \alpha \varsigma)$, debemos demostrar, lo más que se

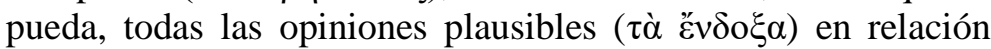
con esas afecciones o, sino las más y las que más dominan, pues

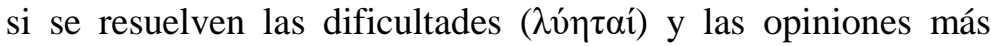
plausibles ( $\tau \alpha \grave{~} \check{v} v \delta o \xi \alpha$ ) queden en pie, se habrá demostrado suficientemente (Ética Nicomáquea VII 1, 1145b 2-7. trad. Sinnot, modificada)

\footnotetext{
${ }^{36}$ De hecho, la edición de Ross compila una serie de fragmentos de esta obra en la cual Aristóteles discute abiertamente esta opinión. El argumento de esta serie de fragmentos (2a- 2f Ross) reviste a grandes rasgos esta forma: si se debe filosofar, se debe filosofar. Sin embargo, si no se debe filosofar, también se debe filosofar. Para un análisis de este argumento, cf. CAStagnoli (2010: 187- 196); HutChinson-Johnson (2018).

${ }^{37}$ Para phainómena adoptamos la traducción de MIE (2013), pues consideramos que, al reproducir la ambigüedad semántica del término griego, se ajusta en mayor medida al texto original.
}

Stylos. 2021; 30 (30); pp. 101-123; ISSN: 0327-8859; E-ISSN: 2683-7900 
Este es quizá uno de los pasajes metodológicos, que, luego de la aparición del trabajo de Owen: "Tithenai Ta Phainomena", 38 ha suscitado mayores dificultades y controversias. Excede a este trabajo introducirnos en esta polémica. ${ }^{39}$ No obstante, el juego de palabras que realiza Aristóteles en este pasaje para hacer referencia al método dialéctico es significativo.

Es clave en primer lugar la equiparación entre phainómena ${ }^{40}$ y éndo$x a$. Como lo indica MIE (2013: 222), el valor epistémico de las opiniones plausibles radica en que los términos "phainómena" y "éndoxa" designan las creencias que usualmente se toman como verdaderas. Dichas creencias, según MIE (2013: 222) "no representan meramente un punto de partida inicial para la investigación, sino que constituyen una configuración conceptual central para llevarla a cabo". ${ }^{41}$

En segundo lugar, es clave la aparición y combinación de diaporésantas, deiknúnai, lúetaí dedeigménon. La alternancia de estos términos nos permite trazar cierto paralelismo entre este pasaje y Metafísica III 1, 995a24-995b4, instancia en la cual el Estagirita describe el método diaporemático. El uso de estos términos delimita dos fases de un mismo proceso: el planteo del problema generado por tesis discrepantes que nos ponen frente una situación aporética (diaporésantas) y la búsqueda de la solución me-

\footnotetext{
38 OWEN (1961).

${ }^{39}$ Para esta cuestión y un análisis minucioso de este pasaje, véase Nussbaum (1982), MiE (2013).

40. Tal como lo recalca, NuSSBAum (1982: 268), phainómena es un término polivalente, razón por la cual, al enfocarse en Ética Nicomáquea VII 1, 1145b 2-7, los estudiosos lo han traducido de diversos modos: "hechos observados", "datos de percepción", "hechos admitidos", "hechos", etc. Sin embargo, han omitido otras posibles maneras de hacerlo, como, por ejemplo, "apariencias", "lo que creemos" o "lo que decimos". Desde la perspectiva de NusSBAUM (1982: 272), estás traducciones tendrían más sentido, en función de que en las líneas siguientes phainómena es sustituido por éndoxa Según NussBAum (1982: 269), en este pasaje Aristóteles usa deliberadamente "apariencia". Al usar esta acepción del término, argumenta Nussbaum, está tomando una posición sobre el método filosófico y sus límites que era muy inusual en la tradición pre-aristotélica, que tendía a contraponer apariencia y realidad, identificando solo a esto último como lo verdadero.

${ }^{41}$ cf. NuSSBAum (1982: 269).
} 
diante la disolución del conflicto (deiknúnai, lúetaí dedeigménon). ${ }^{42} \mathrm{Me}-$ diante este modo de proceder se ponen a prueba las opiniones generalmente admitidas, rescatando lo que hay de certero en ellas, ${ }^{43} \mathrm{o}$ bien demostrando las contradicciones que suponen y, por lo tanto, dejando al descubierto los motivos por los cuales estás deben ser abandonadas. ${ }^{44}$

Según Aubenque, ${ }^{45}$ la razón por la cual Aristóteles procede de este modo radica en que en sus lecciones no sigue ni el orden de la exposición ni al orden de investigación, sino que son una reconstrucción de una investigación realizada con intenciones didácticas. Por tal motivo, señala Aubenque "a veces, tenemos la impresión de que Aristóteles 'problematiza' a efectos pedagógicos una dificultad que ya tiene resuelta". ${ }^{46}$ Como correctamente lo afirma Aubenque y posteriormente Aguirre Santos, ${ }^{47}$ esto no significa que se trate de un simple artificios, sino de un proceder metodológico, que, agregamos nosotros, tiene un inmenso valor didáctico, pues permite que en el proceso de enseñanza el alumno o discípulo no solo realice el mismo proceso de investigación que su maestro, sino que reproduzca, por las razones esgrimidas, el proceso natural de conocimiento. Por tal motivo, podemos concluir, que este proceder metodológico sirve de andamiaje para hacer inteligible aquello que por sí mismo resulta aprehensible para muy pocos hombres. De este modo, Aristóteles busca que sus lecciones: 1) den cuenta de manera

42 Para un análisis pormenorizado de este proceso, véase AguirRe SANTos (2015); Aubenque (2008: 213-259); BOLTON (1990: 185-236); (2003: 237-262); BRUNSChwiG (1990: 237-262); Rossi (2017: 137-159), entre otros.

${ }^{43}$ Un ejemplo claro de ello es Metafísica I 3-9, instancia en la cual Aristóteles analiza en qué medida sus antecesores postularon las cuatro causas propuestas por él en su Física. Si bien, producto de la deliberación (Metafísica I 5, 987 a 2-3), Aristóteles demuestra las falencias que dichas posturas presentan, paralelamente va demarcando en qué sentido estos pensadores pre-anunciaron su propia teoría de las cuatro causas.

${ }^{44}$ Piénsese en Física I 2-4, en donde Aristóteles aborda las creencias de quienes sostienen que hay un solo principio y las de aquellos que consideran que los principios son infinitos. Otro ejemplo de este modo de proceder es Metafísica IV, instancia en la cual Aristóteles da cuenta del principio de no contradicción, analizando las tesis de aquellos que supuestamente lo niegan. En las obras exotéricas este modo de proceder está claramente presente en los fragmentos 16, 17, 19a-c de Sobre la filosofía.

45 AuBENQUe (2008 [1962]:17).

${ }^{46}$ AubenQue (2008 [1962]: 17).

${ }^{47}$ AguirRe Santos (2007: 116).

Stylos. 2021; 30 (30); pp. 101-123; ISSN: 0327-8859; E-ISSN: 2683-7900 
firme de las concepciones presentadas; 2) logren persuadir, de modo tal que los interlocutores estén dispuestos a aceptarlas como discursos verdaderos que debe ser adoptados; y 3) sean eficientes, es decir, se amolde a ciertas pautas cognitivas, sin las cuales lo transmitido difícilmente sea aprendido.

\section{CONCLUSIÓN}

En el presente trabajo hemos intentado analizar el papel de las estrategias dialécticas en los discursos didácticos de Aristóteles. Dado que hemos basado nuestro análisis, tanto en sus lecciones de clases, como en sus discursos exotéricos, en la primera parte nos hemos centrado en la naturaleza de ambos tipos de textos, con el objeto de establecer en qué medida es pertinente el examen de estos últimos para el estudio de los primeros. En este sentido, argumentamos que existe una continuidad temática y doctrinal entre ambos tipos de obras, pero también metodológica. Dado esto, el estudio del modus operandi de Aristóteles en sus obras exotéricas nos permite entender de manera más integral la utilización de la dialéctica en sus lecciones.

En la segunda parte, establecimos que los discursos didácticos deben seguir el orden del conocimiento. Ahora bien, desde la perspectiva de Aristóteles, conocemos algo cuando aprendemos sus principios y causas, razón por la cual el proceso de enseñanza debería adoptar este modo de proceder, esto es, debería ser demostrativo.

Sin embargo, alegamos, reducir el discurso didáctico a esta caracterización tiene dos problemas. El primero es que no es el único modo de proceder que Aristóteles parece seguir en sus lecciones (textos esotéricos) ni en sus discursos exotéricos. El segundo problema es que, si bien posee epistéme en sentido estricto aquel que aprehende las primeras causas y principios, este modo de conocer es propio de algunas almas privilegiadas (Tópicos VI 4, 141b12-14), que, por otra parte, tuvieron que haber alcanzado dicho saber de un modo no demostrativo. Dado esto, incluso en estos casos, en esta particular instancia, el conocimiento parte de lo más "conocido para nosotros" a "lo más conocido en sí" (Física I, 184a16-23; Metafísica VII 3, 1029a 1-4; Ana- 
líticos segundos I 2, 71b 33 y ss, Tópicos VI 4, 142a 6- 12, Sobre la filosofía fragmento 8b, Protréptico fragmentos 102, 105 Düring, 10a2 Rose). Es por este motivo que en sus discursos didácticos y en aquellos que, sin serlo directamente, sigue la misma metodología, Aristóteles se vale de estrategias dialécticas para garantizar el éxito de sus lecciones (Metafísicas II, 3 994b32- 995a3). Bajo esta perspectiva de análisis, podemos afirmar que, si bien ambos tipos de discursos no se identifican (Refutaciones Sofisticas 2 165 1-3), el uso de la dialéctica en sus escritos didácticos obedece a su intento de reproducir el orden de la investigación, de modo tal que el proceso de aprendizaje se produzca de la manera en que solemos conocer naturalmente.

\section{REFERENCIAS BIBLIOGRÁFICAS}

AguirRe SANTOS, J., Dialéctica y filosofía primera. Lectura de la Metafísica de Aristóteles, Zaragoza: Universidad de Zaragoza, 2015.

AguirRe Santos, J. La aporía en Aristoteles: Los Libros B y K 1-2 de La Metafísica, Madrid: Dykinson, 2007.

Aubenque, P., El problema del ser en Aristóteles. trad. cast. de Peña, V., Madrid: Escolar y Mayor Editores, 2008 (1962).

BARNES, J. "Roman Aristotle", in Barnes, 1-70. En Griffin, M. (ed.), Philosophia Togata II: Plato and Aristotle at Rome, Oxford: Claredon Press, 1997.

Berti, E., La filosofia del "primo” Aristotele, Milano, Centro di Ricerche di Metafisica dell’ Università Cattolica del Sacro Cuore, 1997.

Stylos. 2021; 30 (30); pp. 101-123; ISSN: 0327-8859; E-ISSN: 2683-7900 
BignONE, E., L' Aristotele perduto e la formazione filosofica de Epicuro, 2da edizione accresciuta, Firenze: La Nuova Italia, 1973 (1936).

Bolton, R., "The Epistemological Basis of Aristotelian Dialectic", 185236. En Devereux, D. - Pellegrin, P. (Eds.), Biologie, logique et métaphysique chez Aristote, Paris: Éditions Centre National de la Recherche Scientifique, 1990.

Bronstein, D., Aristotle on Knowledge and Learning, The Posterior Analytics, Oxford: Oxford University Press, 2016.

BRUNSCHWIG, J.,"Remarques sur la communication de Robert Bolton”, 237262. En Devereux, D.- Pellegrin, P. (Eds), Biologie, logique et métaphysique chez Aristote, Paris: Éditions Centre National de la Recherche Scientifique, 1990.

Calvo Martínez, T. Aristóteles, Metafísica. Introducción, traducción y notas, Madrid: Gredos, 2007.

Charles, D., "Definition and Explanation in the Posterior Analytics and Metaphysics", 286-322. En Charles, D. (ed), Definition in Greek Philosophy, Oxford: Oxford University Press, 2010.

Castagnoli, L., Ancient Self-Refutation. The Logic and History of the SelfRefutation Argument from Democritus to Augustine, Cambridge: Cambridge University Press, 2015 (2010).

Cleary, J., Aristoteles. Los Múltiples sentidos de prioridad, Trad. castellana de Boeri, M., Buenos Aires: Colihue, 2010 (1988).

Candel Sanmartín, M., Aristóteles. Tópicos en Tratados de lógica I. Introducción, traducción y notas, Madrid: Gredos, 1988.

Candel Sanmartín, M. Aristóteles. Analíticos Segundos, en Tratados de lógica II. Introducción, traducción y notas Gredos, Madrid, 1995.

Stylos. 2021; 30 (30); pp. 101-123; ISSN: 0327-8859; E-ISSN: 2683-7900 
DÜRING, I. Aristotle's Protrepticus. An Attempt at Reconstruction, Göteborg: Almqvist \& Wiksell, 1961.

DÜRING, I. Aristóteles. Exposición e interpretación de su pensamiento. Trad. cast. de Navarro, B. México: Universidad Nacional Autónoma de México, 2005 (1966).

DuRING, I. Aristotle in the Ancient Biographical Tradition, Gúteborg: Almqvist \& Wiksell Stockholm, 1957.

Echandía, G., Aristóteles. Física. Introducción, traducción y notas, Madrid: Gredos, (1995).

HATZIMICHALI, M. "The texts of Plato and Aristotle in the first century B. C", 1-27. En Schofield. M. (ed) Aristotle, Plato and Pythagoreanism in the First Century B.C., Cambridge: Cambridge University Press, 2012.

Hutchinson D. S. - Johnson, M. R. "Protreptic and Apotreptic: Aristotle's Dialogue Protrepticus",111-154. En, Kotzé, A. - Van der Meeren, S. (ed.), When Wisdom Calls Philosophical Protreptic in Antiquity, Turnhout: Brepols, 2018.

JAEGER, W. Aristóteles. Bases para la historia de su desarrollo. Trad. cast. Xirau, J. - Wenceslao Roces, W. México: Fondo de Cultura Económica, 1993 (1923).

LLOYD, G. E. R., Aristotle: The Growth and Structure of his Thought, Cambridge: Cambridge University Press, 1968.

MiÉ, F., "Dialéctica y ciencia en Aristóteles", Signos Filosóficos, 2009 XI 21, 9-42.

Stylos. 2021; 30 (30); pp. 101-123; ISSN: 0327-8859; E-ISSN: 2683-7900 
MiÉ, F., "Phenomena and Beliefs in Aristotle. An interpretation on the methodological role of éndoxa in natural science", LOGOS. Anales del Seminario de Metafísica, 2013 46, 211-234.

MorauX, P., Le Listes Anciennes des ouvrages d'Aristote, Louvain: Éditions, Universitaires de Louvain, 1951.

Nussbaum, M. C. “Saving Aristotle's appearances”, 267-294. En Schofield, M. (ed.), Language and Logos Studies in ancient Greek philosophy presented to G. E. L. Owen, Cambridge: Cambridge University Press, 1982.

Owen, G. E. L. "Tithenai ta phainomena", 83- 103. En Aristote et les problèmes de méthode, Louvain, 1961.

PAJÓn LeYRA, I. "The Aristotelian Corpus and the Rhodian Tradition: New Light from Posidonius on the Transmission of Aristotle's Works", The Classical Quarterly 2013, 63723 - 733.

RAPP, C. "The Nature and Goals of Rhetoric", 579-596. En Anagnostopoulos, G. (ed.), A Companion to Aristotle, Oxford: Blackwell Publishing, 2009.

Rose, V. Aristotelis qui ferebantur Librorum Fragmenta, Teubner: Stuttgart, 1966 (1886).

Ross, W. D. Aristotelis Dialogorum Fragmenta, Oxford: Clarendon Press, 1964.

RossI, G. "Going through Aporiai: The Critical Use of Aristotle's Dialectic", Oxford Studies, Ancient Philosophy, 2017, 209 - 256.

Seggiaro, C. "El fragmento 48 del Protréptico de Aristóteles: ¿vocabulario platónico o concepciones platónicas?”, Revista Classica, 2016 29, n. 2, 33-54.

Stylos. 2021; 30 (30); pp. 101-123; ISSN: 0327-8859; E-ISSN: 2683-7900 
SINNOTT, E. Aristóteles. Ética Nicomáquea. Introducción, traducción y notas, Buenos Aires: Colihue, 2015.

TANNER, R. G. Aristotle's Works: The Possible Origins of the Alexandria Collection, 1-15. En MacLeod, R. (ed.), The Library of Alexandria, London: I.B.Tauris, 2010.

VALlEJo CAMPOS, A. Aristóteles. Fragmentos. Introducción, traducción y nota, Gredos: Madrid, 2005

Stylos. 2021; 30 (30); pp. 101-123; ISSN: 0327-8859; E-ISSN: 2683-7900 